

\section{TRANSICIÓN ENERGÉTICAARGENTINA. EL NUEVO ESTÁNDAR DE EFICIENCIA ENERGÉTICA EN LA EVALUACIÓN DE LA VIVIENDA SOCIAL. CASO DE ESTUDIO: VIVIENDA DE BARRIO PAPA FRANCISCO}

\section{ARGENTINEAN ENERGY TRANSITION. THE NEW ENERGY EFFICIENCY STANDARD IN THE EVALUATION OF SOCIAL HOUSING. CASE STUDY: DWELLING IN PAPA FRANCISCO NEIGHBORHOOD}

\author{
ERNESTO KUCHEN \\ Doctor en Arquitectura \\ Investigador CONICET - Subdirector del Instituto Regional de \\ Planeamiento y Hábitat. Docente e Investigador Facultad de \\ Arquitectura Urbanismo y Diseño \\ Instituto Regional de Planeamiento y Hábitat - Consejo Nacional \\ de Investigaciones Científicas y Técnicas (CONICET) - Universidad \\ Nacional de San Juan (IRPHa-UNSJ-CONICET) \\ San Juan, Argentina \\ http://orcid.org/0000-0001-6649-6932 \\ ernestokuchen@faud.unsj.edu.ar
}

\author{
DANIEL KOZAK \\ $\mathrm{PhD}$ in Built Environment \\ Investigador CONICET - Director del Centro de Investigación Habitaty \\ Energía. Universidad de Buenos Aires (CIHE-FADU-UBA) \\ Centro de Estudios Urbanos y Regionales (CEUR)-Consejo Nacional \\ de Investigaciones Científicas y Téenicas (CONICET)-Centro de \\ Investigación Habitat y Energía (CIHE-FADU-UBA) \\ Buenos Aires, Argentina \\ https://orcid.org/0000-0003-3118-8950 \\ daniel.kozak@conicet.gov.ar
}

\section{RESUMEN}

El sector edilicio es responsable del $40 \%$ de la demanda energética a nivel internacional y el $37 \%$ en Argentina. La climatización constituye el ítem más representativo. Los países instituyen medidas para el uso racional de la energía y persiguen estrategias a fin de provocar la transición energética. La aprobación del estándar IRAM 11900 en Argentina promueve la Eficiencia Energética (EE) en el sector edilicio residencial que se pretende, sea inclusivo y conduzca a la transición al 2050. El objetivo del trabajo es la evaluación

termo-energética de un prototipo existente de vivienda de interés social del Barrio Papa Francisco

y sus variantes mejoradas "retrofit" y "ex ante", desde el enfoque de la norma. Para ello se relevan dimensiones, sistemas constructivos, componentes de la envolvente y equipamiento para cubrir demandas de calefacción, refrigeración, agua caliente sanitaria e iluminación. Como resultado relevante, el índice de prestaciones energéticas (IPE) del prototipo existente alcanza un valor de $132 \mathrm{kWh} \mathrm{prim}_{\text {. }} / \mathrm{m}^{2}$.año. Además, las variantes mejoradas "retrofit" y "ex ante" conducen a ahorros económicos considerables en la inversión inicial. De aquí que se valora la importancia de implementar software de análisis en la etapa de proyecto para cuantificar los recursos energéticos y el ahorro de emisiones en una transición energética planificada.

\section{Palabras clave}

indice-ipe, vivienda social, norma iram-11900:2017, etiquetado energético

\section{ABSTRACT}

The building sector is responsible for $40 \%$ of the energy demand internationally, and for $37 \%$ in Argentina. Heating and cooling are the most representative item. Rational energy usage measures are being introduced in countries throughout the world, as well as strategies that pursue energy transition. The passing of the IRAM 11900 standard in Argentina promotes energy efficiency (EE) in the residential building sector, and it is expected to be inclusive and lead to the transition by 2050. The aim of this work is to perform the thermo-energy evaluation of an existing social dwelling prototype in "Barrio Papa Francisco" (Pope Francisco Neighborhood), as well as in its two improved variations: "retrofit" and "ex ante". For this, information is collected about the dimensions, construction systems, building envelope components and equipment to meet demands for heating, cooling, domestic hot water and lighting. As a relevant result, it is reported that the energy supply index (IPE, in Spanish) of the existing prototype reaches a value of $132 \mathrm{kWh}$ prim $/ \mathrm{m}^{2}$.year. In addition, the retrofit and ex ante variations lead to considerable economic savings in the initial investment. This is why the importance of implementing software analysis at the design stage is considered as important in order to quantify energy resources and emissions savings in a planned energy transition. 


\section{INTRODUCCIÓN}

A nivel internacional las acciones para la transición energética difieren según las economías y estructuras sociales de los países. Modelos STET, Socio-Technical Energy Transition (en español, transición energética socio-técnica), permiten detectar focos y dinámicas que orientan a los países en sus transiciones, poniendo en discusión la participación tecnológica en sus economías y los valores de demanda de energía objetivo y emisiones de carbono (Li, Trutnevyte y Strachan, 2015). Las economías emergentes priorizan cambios de empleo de biomasa por vectores energéticos modernos. Las estrategias en países desarrollados, como Reino Unido, Francia y Holanda, van desde modelos de "retrofit" para la envolvente edilicia en la conservación de la energía, pasando por la planificación "ex ante" de la eficiencia energética, hasta modelos de desarrollo territorial para el crecimiento sustentable de nuevos barrios. Alemania, pionera en transición energética, desarrolla hace casi dos décadas el programa ENOB, Energie Optimiertesbauen (en español, Construcción Energéticamente Optimizada), que se centra en lograr una demanda de energía primaria para iluminación, calefacción/enfriamiento y ventilación de $100 \mathrm{kWh}$ Prim $/ \mathrm{m}^{2} \mathrm{a}$, en base a modelos planificados ex ante (Kuchen, Plesser y Fisch, 2012). Hoy, avanza con el programa ENERGIE WENDE BAUEN 2020 (Programa para la Transición Energética en Construcción) ${ }^{1}$, del Ministerio de Economía y Energía, Ilamado Energieoptimierte und klimaneutrale Gebäude der Zukunft (Planificación de edificios con eficiencia energética e impacto ambiental neutro), impulsando modelos de proyectos demostrativos de baja energía primaria, con renovables, y reducción de emisiones en las 4 fases de la vida útil.

La transición argentina persigue al 2050 la diversificación del mix energético con renovables, Eficiencia Energética (EE) en la vivienda, electrificación de la energía final, la digitalización de datos y el cambio de estrategias para bajar emisiones en industria, silvicultura y ganadería (Fernández, 2019); un plan que reúne los aportes de la experiencia internacional. El sector residencial, responsable de casi el $80 \%$ del consumo de recursos dentro del sector edilicio por la demanda calórica (Chevez, 2017), además de presentar el mayor potencial de acción, queda sujeto a constituirse en el talón de Aquiles en la transición, debido a su diversidad y extensión (Riavitz, Zambon y Giuliani, 2015).

El Programa Nacional de Etiquetado de Viviendas en Argentina tiene como objetivo introducir la Etiqueta de EE como un instrumento que brinde información a los usuarios acerca de las prestaciones energéticas de la vivienda (Alonso Frank y Kuchen, 2017). El estándar, con acento en modelos de abordaje de situaciones ex ante y retrofit, pretende generar, además, valor agregado a inmuebles (Secretaría de Energía de la Nación SEN, 2020). El etiquetado tiene por fin constituirse en herramienta de decisión al realizar, con conciencia ambiental, una operación inmobiliaria: evaluar un nuevo proyecto o intervenir en viviendas existentes (SEN, 2020).

El método para obtener el índice de prestaciones energéticas (IPE), normalizado en el estándar de referencia (IRAM 11900, 2017), representa el requerimiento de "energía primaria" por unidad de superficie y año [kWh $\mathrm{kPrim}_{\text {. }}$ $\mathrm{m}^{2}$.año], para satisfacer necesidades de calefacción, refrigeración, agua caliente sanitaria e iluminación de una vivienda. Según Risuelo (2010), el etiquetado debería suministrar información sobre diversas medidas e inversiones a nivel tecnológico, de gestión y de hábitos culturales de los ciudadanos.

Las resoluciones iniciales desde la concepción de los proyectos son las más determinantes y las que ofrecen un mejor balance en la relación costo-beneficio. Resolver correctamente la orientación de un edificio, el grado de compacidad, dimensionar superficies vidriadas en base a las características climáticas del emplazamiento, ceteris paribus, no debería resultar más oneroso que las prácticas negligentes; además de que cualquier corrección durante la marcha de la obra suele ser extremadamente difícil (Kozak, Evans, Adamo, Abálsamo y Romanello, 2017).

Del mismo modo, la elección de materiales estructurales y operaciones de diseño sobre aberturas, ubicación de los filos y retranqueos de la superficie vidriada, tienen fuerte incidencia en la ecuación de costos económicos versus los beneficios energético-ambientales y de confort térmico (Evans, De Schiller y Kozak, 2015).

A medida que avanza el proyecto y construcción, se van cerrando oportunidades y para lograr un rendimiento termo-energético equivalente debe invertirse más dinero. Lo mismo ocurre con decisiones que atañan a la sustentabilidad, como la elección de un material según su impacto ambiental a partir de la energía embebida en su producción y la utilizada durante su traslado, o el grado de sanidad ambiental del proceso industrial durante su fabricación (Brent y Petrick, 2007).

En este trabajo se presenta el estudio de determinadas alternativas de estrategias constructivas, posibles de implementarse en una transición energética nacional, en el que se evalúa costos económicos y rendimiento. Como regla general, cuanto antes se incorporen las estrategias de EE y sustentabilidad en un proyecto de arquitectura, se conseguirán mayores beneficios a menores costos. 


\section{METODOLOGÍA}

En términos metodológicos, en primer lugar, se identifica una unidad representativa del Barrio Papa Francisco. Luego, se lleva a cabo un trabajo de campo para la recolección de datos a analizar. Se relevan características geo-referenciales, técnico-constructivas (muro, solado, cubierta y abertura), adyacencias, obstrucciones solares, zonas térmicas, ambientes no climatizados o no habitables, así como equipamiento destinado a cubrir demandas de calefacción, refrigeración, agua caliente sanitaria, iluminación y aporte renovables. Para la evaluación de las prestaciones energéticas de la unidad, se consideran las exigencias del estándar (IRAM 11900: versión 2017) y para el cálculo, el aplicativo informático (software) de referencia (Etiquetado de Viviendas). En función de los resultados obtenidos de la unidad de referencia, se practican mejoras a los componentes constructivos de la envolvente opaca y transparente en las instancias retrofit, es decir, la adecuación retroactiva de la vivienda terminada, y ex ante, o sea, simulando la etapa de anteproyecto previo a la construcción de la vivienda. Además de las ventajas energéticas de abordar diferentes estrategias, en un proceso conducente hacia una transición energética aplicada mediante este tipo de modelos, se evalúan las conveniencias económicas y ambientales.

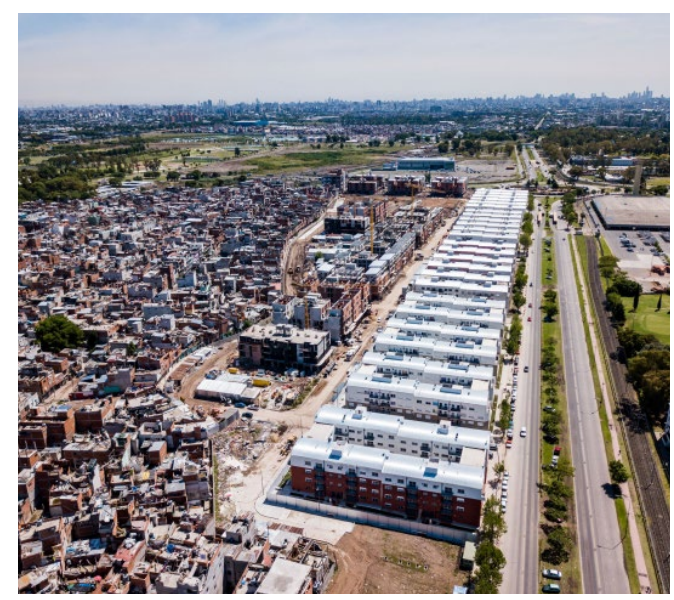

a) Avance obra al año 2019.

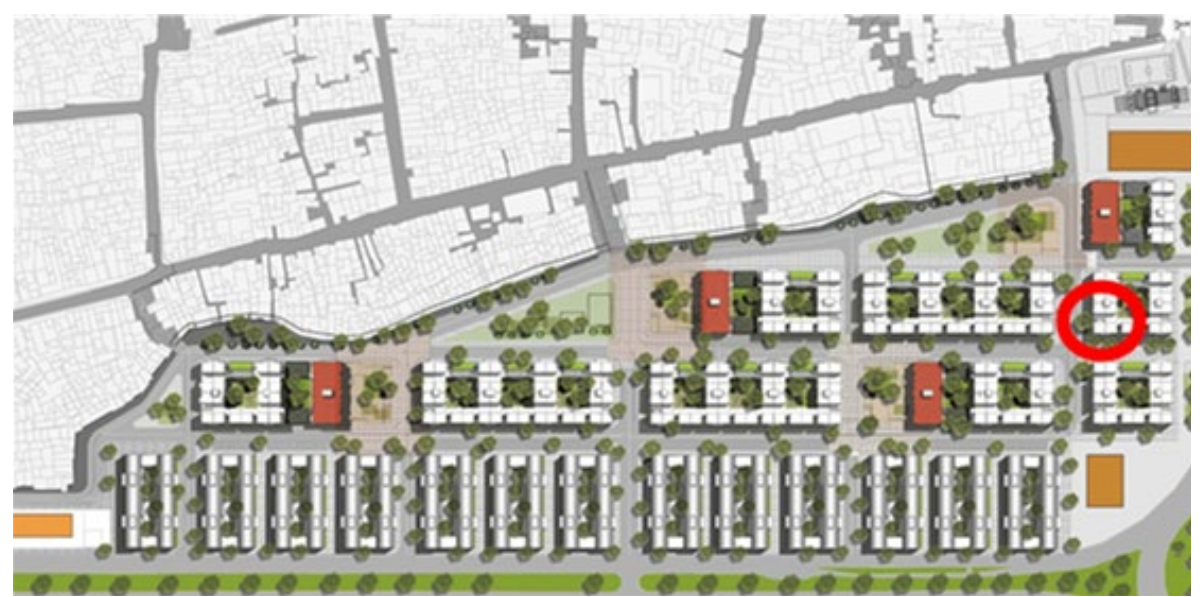

b) Emplazamiento del objeto de estudio.

Figura 1. Intervención de vivienda de interés social en Barrio Papa Francisco.

Fuente: Instituto de la Vivienda de la Ciudad (IVC). Buenos Aires.

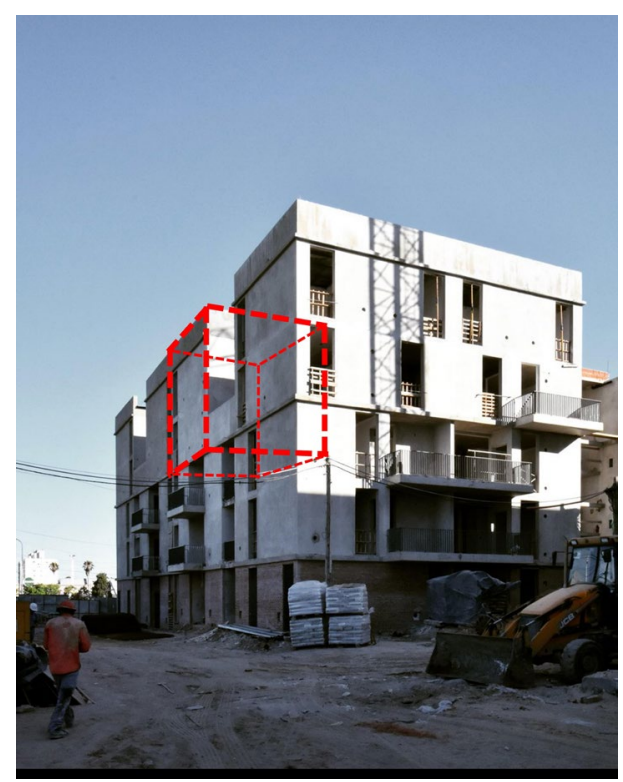

a) Identificación de la unidad en línea de trazo discontinua (en rojo).

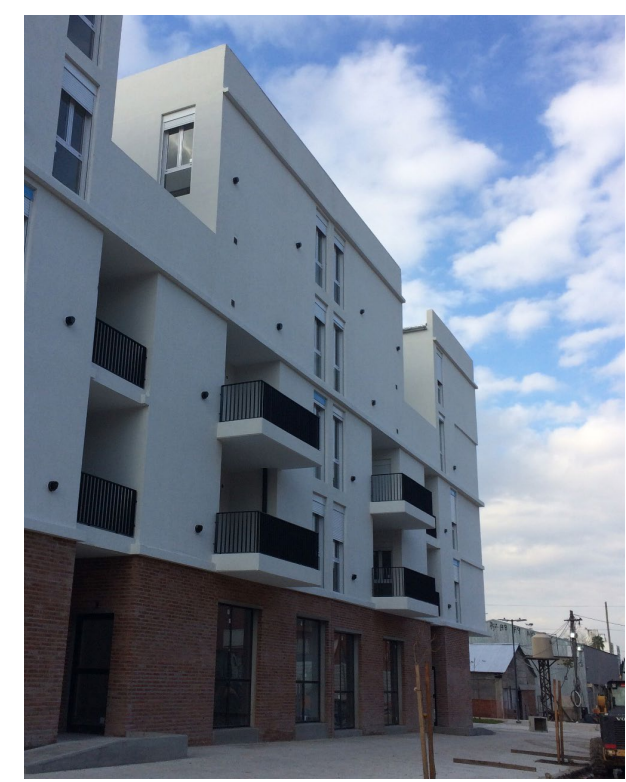

b) Terminación fachada y zócalo comercial en PB.

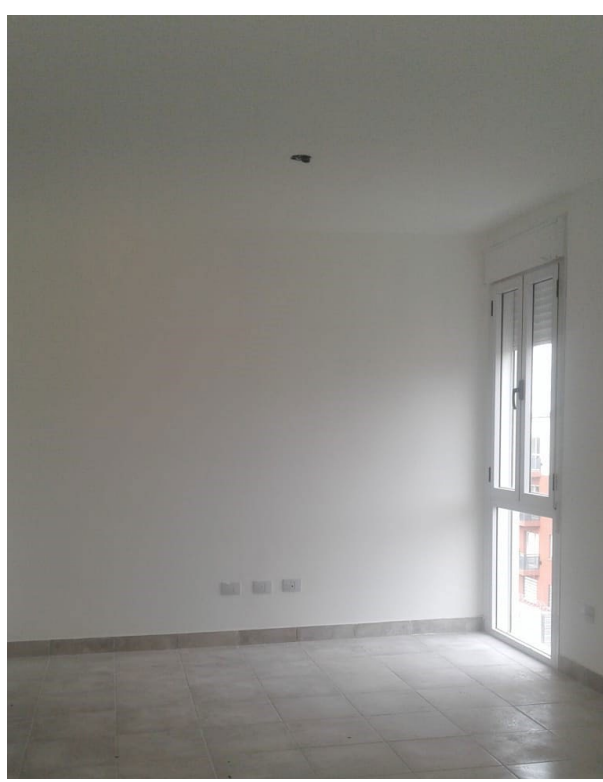

c) Acabado superficial interior y abertura de aluminio. 


\section{IDENTIFICACIÓN DEL CASO DE ESTUDIO}

En Villa 20 viven 27.000 personas, en 4.581 viviendas (48 hectáreas). La propuesta de "integración de los barrios" (Instituto de la Vivienda de la Ciudad - IVC, 2016) consiste en generar 1.700 nuevas unidades habitacionales (Figura 1a). Se elige un prototipo existente como caso de estudio en vivienda social de la relocalización de Villa N²0 a Barrio Papa Francisco, en Villa Lugano, Provincia de Buenos Aires, en zona bioambiental III-B, con clima templado cálido húmedo, según clasificación de norma IRAM 11603 (2012), con temperatura media de invierno de $12,8^{\circ} \mathrm{C}$ (mín. de $9,7^{\circ} \mathrm{C}$ y máx. de $16^{\circ} \mathrm{C}$ ) y de verano, de $23,3^{\circ} \mathrm{C}$ (mín. de $19,6^{\circ} \mathrm{C}$ y máx. de $27^{\circ} \mathrm{C}$ ), y humedad relativa ambiente del $77 \%$, en invierno, y $69,3 \%$, en verano. La demanda de energía de calefacción en base de confort $20^{\circ} \mathrm{C}$, según IRAM 11603 (2012), es de 1249 grados/día. El prototipo de "tipología compacta" es estándar, dentro del conjunto de otros con similares características (Figura 1b).

\section{CARACTERÍSTICAS TÉCNICO-CONSTRUCTIVAS}

El inmueble de estudio se sitúa en el $3^{\text {er }}$ piso de un bloque, al que se accede por escalera y que se agrupa en 8 subbloques de 5 niveles cada uno (Figura 2a), con zócalo comercial en Planta Baja y departamentos simplex en $1^{\text {er }} y$ $2^{\text {do }}$ nivel (Figura $2 b$ ).

El prototipo es un dúplex con superficie útil de $78,6 \mathrm{~m}^{2}$, altura interior de 2,6 m (Figura 2c) y volumen de $204,5 \mathrm{~m}^{3}$. El

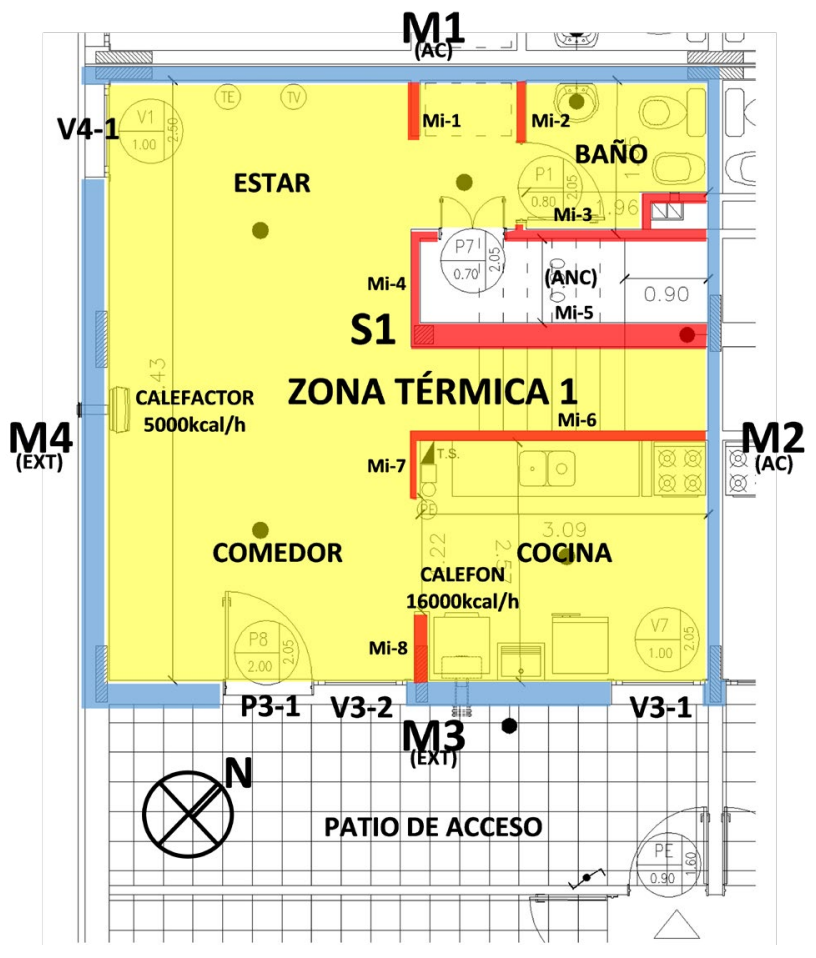

a) Planta baja del prototipo en dúplex. programa se compone de patio de acceso exterior a estarcomedor, cocina y baño en planta baja y 3 dormitorios con baño completo en planta alta (Figura 3).

La envolvente se compone de muros de bloque cerámico hueco de $18 \mathrm{~cm}$, con revoque exterior termoaislante del tipo Isolteco de $3 \mathrm{~cm}$, color blanco, revoque interior plástico tipo revear de $2 \mathrm{~cm}$ (blanco), con coeficiente de transferencia $K=1 \mathrm{~W} / \mathrm{m}^{2} \mathrm{~K}$; cubierta de chapa galvanizada, aislada con $5 \mathrm{~cm}$ de lana de vidrio y cielorraso con placa de yeso de $1,5 \mathrm{~cm}$ (blanco), $K=0,72 \mathrm{~W} / \mathrm{m}^{2} \mathrm{~K}$ y solado de cerámico (color beige) $K=2,9 \mathrm{~W} / \mathrm{m}^{2} \mathrm{~K}$ (entrepiso). Las aberturas, de $1 \mathrm{~m}$ de ancho y 2,10 $\mathrm{m}$ de altura son de abrir, aluminio blanco, doble contacto y vidrio simple. Como protección solar poseen cortinas de enrollar de PVC blanca, con tapa rollo sobre marco superior hasta altura de dintel (Figura 2c), $K=3,68 \mathrm{~W} / \mathrm{m}^{2} \mathrm{~K}$.

\section{EQUIPAMIENTO}

Destinado a cubrir requerimientos para asegurar calidad de vida, mantener la temperatura interior $>20^{\circ} \mathrm{C}$ en invierno y $<26^{\circ} \mathrm{C}$ en verano, agua caliente $\left(42^{\circ} \mathrm{C}\right)$ e iluminación en ambientes interiores $>300$ Lux. Para calefacción, tiene una potencia instalada de $5,82 \mathrm{~kW}$, en calefactores tiro balanceado, 1 de $5000 \mathrm{kcal} / \mathrm{h}$ y 3 de $2500 \mathrm{kcal} / \mathrm{h}$. Para Agua Caliente Sanitaria (ACS), un calefón de $16000 \mathrm{kcal} / \mathrm{h}$ (ver Figura 3), en iluminación, lámparas LED con un total de $72 \mathrm{~W}$. No posee instalación para refrigeración. No presenta Energías Renovables.

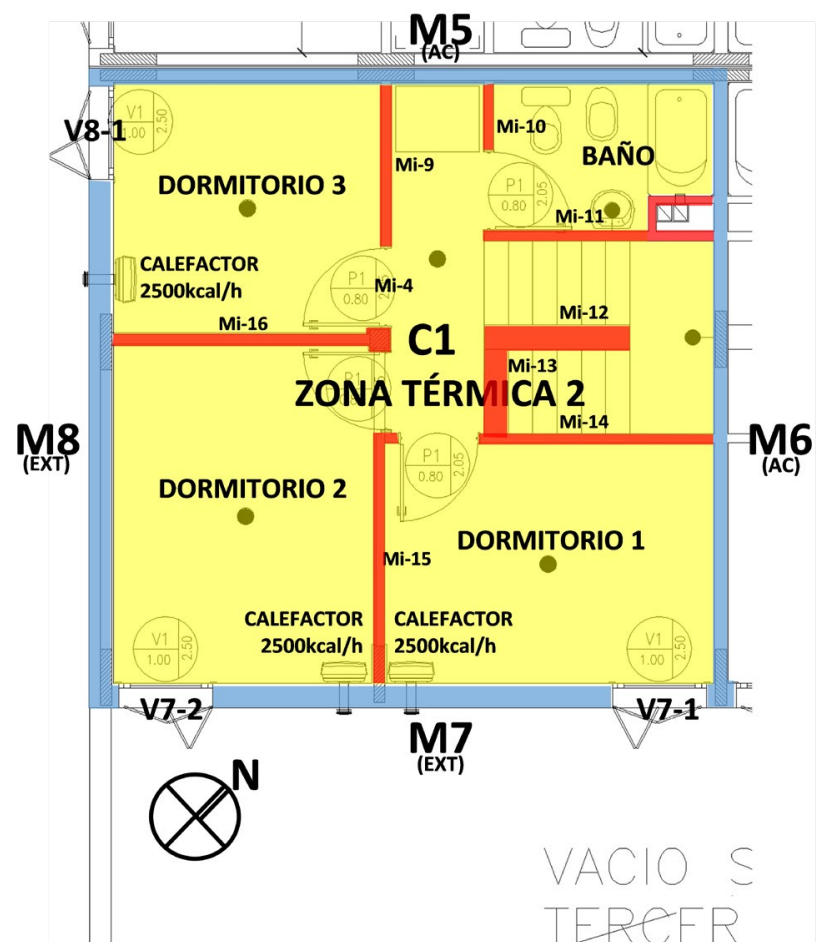

b) Planta alta del dúplex. 


\section{RESULTADOS Y DISCUSIÓN}

Mediante la implementación del software aplicativo de cálculo (Etiquetado de Viviendas, 2020) que promueve la Secretaría de Energía de la Nación (SEN, 2020), según exigencias de la norma (IRAM 11900, 2017), se calculan características dinámicas, requerimientos e indicadores del comportamiento térmico-energético a fin de analizar variantes de decisión para la eficiencia y el saneamiento energético de viviendas.

\section{CARACTERÍSTICAS ENERGÉTICAS DINÁMICAS}

Las características energéticas dinámicas se refieren a la relación entre los aportes y las pérdidas de cada período en que se demanda energía adicional. A partir de la simulación, se detecta que, del total de los aportes gratuitos, se logra captar el 30\% en invierno y es posible aprovechar casi el total de los mismos (97\%). Inversamente a esto, el porcentaje de pérdidas por ventilación y conducción de energía térmica desde la envolvente en verano (dispersión térmica), es del $48 \%$ de lo que solo es aprovechable, menos de la mitad (47\%). El emplazamiento del prototipo presenta condiciones desfavorables de asoleamiento en relación a los "aportes gratuitos", y de ventilación efectiva para la "dispersión térmica".

El grado de compacidad del inmueble puede ser beneficioso. En este, la relación entre área de envolvente

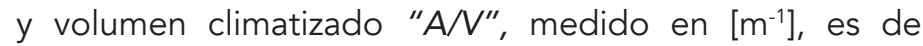
$1,5 \mathrm{~m}^{-1}$ (buena). A este beneficio, se le suma el "factor de intercambio térmico", que hace referencia a la ubicación de la unidad respecto del conjunto, con valor adimensional en escala de 0-1, siendo de 0,36 (bajo). De ello, se evidencia que la ubicación aporta un beneficio en invierno. La estrategia de ventilación de la vivienda juega un rol fundamental en la "dispersión térmica", debiendo asegurarse en verano, sobre todo en horas de la noche cuando la temperatura exterior disminuye, por ventilación cruzada.

El "factor de intercambio térmico" se refleja en el valor del "coeficiente global de intercambio térmico $(H)$ ", que indica que por cada grado de diferencia de temperatura entre interior-exterior deberá asegurarse un requerimiento de energía según estaciones del año. Para este prototipo, $\mathrm{H}$ es $106 \mathrm{~W} / \mathrm{K}$ en invierno (bajo) y de 2,6 veces mayor en verano, $274 \mathrm{~W} / \mathrm{K}$ (alto). Un coeficiente $\mathrm{H}$ bajo indica mejores niveles de "constante de tiempo", medido en horas [h], es decir la capacidad térmica de la envolvente para absorber el salto térmico interior-exterior, siendo $28,5 \mathrm{~h}$ para invierno y $11 \mathrm{~h}$ para verano.

\section{ÍNDICE DE PRESTACIONES ENERGÉTICAS (IPE)}

El IPE representa el valor de energía primaria $\left[k W h_{\text {Energia }}\right.$ Primaria $/ \mathrm{m}^{2}$. a] en referencia al impacto ambiental del uso de la energía demandada y su valor da cuenta del nivel de eficiencia energética. En relación al prototipo evaluado, la

\begin{tabular}{|c|c|c|c|}
\hline $\begin{array}{c}\text { Tipo de Energía medida en [kWh } \\
\text { / m2 . año] }\end{array}$ & Útil & Neta & Primaria \\
\hline Calefacción & 47 & 72 & 90 \\
\hline Refrigeración & 12 & 5 & 18 \\
\hline Producción ACS & 12 & 16 & 20 \\
\hline Iluminación & - & 1 & 4 \\
\hline Requerimiento específico global de energía & 132 \\
\hline $\begin{array}{c}\text { Contribución específica de } \\
\text { energías renovables }\end{array}$ & 0 \\
\hline $\begin{array}{c}\text { Índice de Prestaciones Energéticas, } \\
\text { IPE. [kWh / m2. año] }\end{array}$ & 132 \\
\hline
\end{tabular}

Tabla 1. Total de energía requerida para cubrir demandas térmicas y lumínicas de la vivienda. Fuente: Elaboración de los autores.

Tabla 1 muestra valores de "energía útil, neta y primaria" que se debe entregar al sistema, por $\left[\mathrm{m}^{2}\right]$ útil del inmueble en un año. El IPE resultante en Tabla 1, de $132 \mathrm{kWh}_{\text {Prim. }}$ I $\mathrm{m}^{2}$.a, es un valor de referencia del requerimiento de energía primaria y que en relación al promedio, en la etiqueta se corresponde con la Categoría D (color amarillo), es decir, "calidad media o estándar" según IRAM 11900:v.2017.

La "energía útil" es la energía térmica que permitirá mantener la temperatura interior $\geq 20^{\circ} \mathrm{C}$ en invierno con Calefacción $\left(47 \mathrm{kWh} / \mathrm{m}^{2} . \mathrm{a}\right), \leq 26^{\circ} \mathrm{C}$ en verano con Refrigeración (12 kWh/m².a) y $\geq 42{ }^{\circ} \mathrm{C}$ el ACS (12 kWh/ $\mathrm{m}^{2}$.a). Estos valores de energía encontrados para este inmueble de $78,6 \mathrm{~m}^{2}$, variarán según zona climática, forma, asoleamiento, compacidad, infiltraciones y aislación, entre otros. La "energía neta" representa la energía que requiere la vivienda para cubrir la demanda térmica y lumínica (eléctrica y/o gas). Este indicador permite ver reflejado el rendimiento de los equipos instalados. La "energía primaria" es la energía que se necesita obtener, antes de ser transformada y/o transportada, para ser entregada como energía térmica o lumínica al ambiente. Para calcularse se emplean "factores de reducción a energía primaria" y su valor dependerá del tipo de matriz energética. Para el caso de Argentina, el factor de reducción del vector gas a energía primaria será de 1,25 y de electricidad de 3,3.

\section{CALEFACCIÓN}

La calefacción "calefactor gas de tiro balanceado" se refleja al observar que, para entregar $47 \mathrm{kWh} \mathrm{Util}_{\mathrm{it}} / \mathrm{m}^{2}$.a, hace falta una cantidad adicional de energía que se pierde por ventilación de los gases de combustión. Se deberá consumir $72 \mathrm{kWh}_{\mathrm{Neta}} / \mathrm{m}^{2}$.año (ver Tabla 1), es decir, un 65\% más, sin obtener el beneficio térmico. En el caso de tratarse del vector "gas distribuido por redes", hace falta disponer de $90 \mathrm{kWh}$ Prim. $/ \mathrm{m}^{2}$.a, para cubrir la demanda térmica, es decir, un $25 \%$ adicional sobre la energía neta entregada. 


\section{REFRIGERACIÓN}

La unidad estudiada no posee equipamiento de refrigeración, lo que no quiere decir que no lo necesite. Según cálculos para mantener el interior $\leq 26^{\circ} \mathrm{C}$, se requieren $12 \mathrm{kWh} \mathrm{Util}_{\mathrm{l}} / \mathrm{m}^{2}$.año. Condiciones térmicas no adecuadas llevarían al usuario a instalar, por ejemplo, un Split. El coeficiente de performance COP, de clase " $A$ " de $E E$, en Argentina es $C O P=3,3$; lo cual significa que por cada unidad de energía eléctrica entrega 3,3 unidades de energía térmica. Al no conocerse el COP, el aplicativo provee un valor hipotético de $\mathrm{COP}=2,4$ (Categoría $\mathrm{C}$ $\circ$ D), requiriendo $5 \mathrm{kWh}_{\mathrm{Neta}} / \mathrm{m}^{2}$. año (ver Tabla 1). Para el abastecimiento de energía eléctrica se requieren 18 $\mathrm{kWh}_{\text {Prim. }} / \mathrm{m}^{2}$.año.

\section{ACS}

La energía térmica útil que hay que agregarle al agua para elevarla a $42^{\circ} \mathrm{C}$ es de $12 \mathrm{kWh}$ Util. $/ \mathrm{m}^{2}$.a, valor que depende de la superficie útil de la vivienda y variará en función del rendimiento del equipo. Un calefón a gas con encendido instantáneo y piloto automático será un $30 \%$ más eficiente que el que mantiene el piloto encendido. La potencia instalada en producción de ACS (16000 kcla/h) cubre la demanda de energía útil en base al sistema convencional que requiere $16 \mathrm{kWh} \mathrm{Neta}_{\text {. }} / \mathrm{m}^{2}$.año (un $30 \%$ más) y que, por abastecerse de gas de red, requerirá un 25\% adicional, esto es, $20 \mathrm{kWh}$ Prim. $/ \mathrm{m}^{2}$.año.

\section{ILUMINACIÓN}

La tecnología LED permite transformar casi la totalidad de la energía neta en útil, aunque el impacto del uso de la red de electricidad seguirá siendo alto, ya que para entregar de $1 \mathrm{kWh} \mathrm{Neta.} / \mathrm{m}^{2}$.año se requerirán $4 \mathrm{kWh} \mathrm{h}_{\text {Prim.. }} / \mathrm{m}^{2}$.año.

\section{ENERGÍAS RENOVABLES}

La vivienda no dispone de energías renovables. El supuesto de incorporar estas tecnologías (solar, eólico, biomasa, geotérmico, etc.), implica que la fracción generada para autoconsumo térmico o eléctrico reducirá la demanda total de energía primaria.

\section{REQUERIMIENTO TOTAL DE ENERGÍA (GAS Y ELECTRICIDAD)}

Del requerimiento total de energía de los vectores electricidad $394 \mathrm{kWh}$ Prim. /año y gas $4187 \mathrm{kWh}_{\text {Prim. }}$ / año, el de mayor demanda es por calefacción 2944 $\mathrm{kWh}_{\text {Prim }}$ /año y ACS $1243 \mathrm{kWh}_{\text {Prim }}$ /año. La demanda de Refrigeración alcanza 299 kWh Prim $/$ año y la de iluminación es despreciable.

\section{REQUERIMIENTO TOTAL DE ENERGÍA SEGÚN ZONAS TÉRMICAS}

Por tratarse de un dúplex, se se deben identificar dos zonas térmicas (Figura 3): Zona 1 (Z1) en Planta Baja y Zona 2 (Z2) en Planta Alta. No presentan diferencias significativas de demanda térmica de calefacción en invierno. Z1 tiene un requerimiento de energía de 1779 $\mathrm{kWh}_{\text {Util }} / \mathrm{a}$ y Z2 de 1914 kWh $\mathrm{Util}_{\mathrm{l}} / \mathrm{a}$. En verano, la dispersión térmica no llega a ser efectiva siendo de $287 \mathrm{kWh}_{\text {Util }} / \mathrm{a}$ en Z1 y más del doble en Z2, alcanzando 687 kWh Util $_{\text {lail }}$. Esta diferencia se puede atribuir al bajo nivel de aislación y elevado grado de exposición de la cubierta.

\section{COMPORTAMIENTO DE LA ENVOLVENTE}

Las diferencias energéticas observadas en las zonas Z1 y Z2 conducen a realizar una evaluación particular de la envolvente con sus componentes opacos: muro

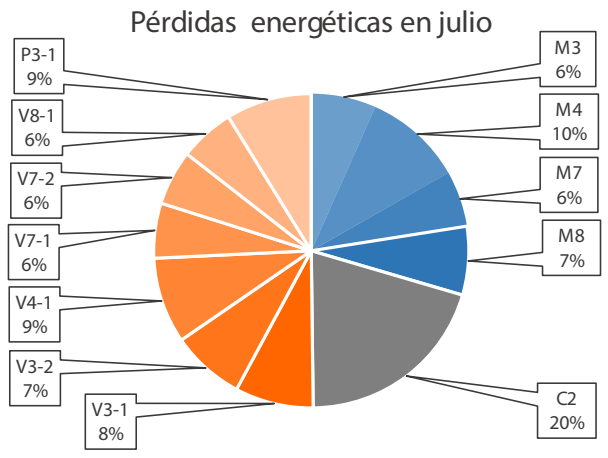

a) Pérdidas
Ganancias energéticas de enero

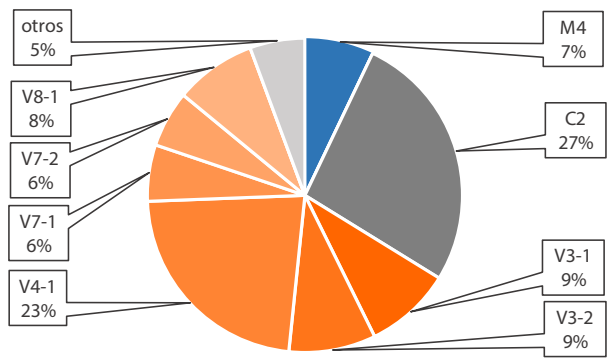

b) Ganancias 
PERDIDAS ENERGÉTICAS JULIO

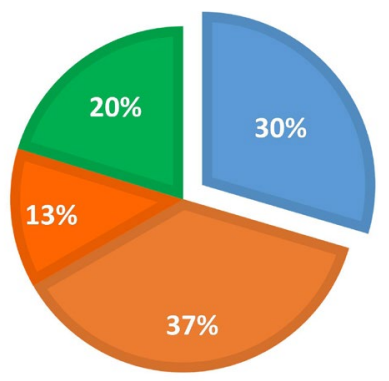

GANACIAS ENERGÉTICAS ENERO

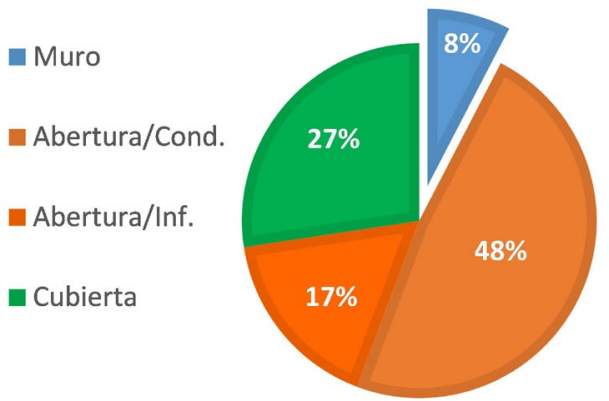

Figura 5. Pérdidas y ganancias por la envolvente, según meses de julio y enero. Fuente: Elaboración de los autores.

$(M)$, solado $(S)$ y cubierta $(C)$; aberturas: ventana $(V)$ y puerta $(P)$. La Figura 4 muestra la influencia porcentual de las partes constructivas, en relación al requerimiento energético en los períodos de mayor demanda mensual: julio, por pérdidas (calefacción), y enero, por ganancias (refrigeración).

De la sumatoria de pérdidas y ganancias equivalentes, es en particular la cubierta (C2) la que se muestra como la más importante, con pérdidas en julio equivalentes al $20 \%$ y ganancias en enero del $27 \%$ del total. Además, el muro (M4) corresponde al de mayor pérdida en julio $y$, sobre el mismo, la abertura (V4-1), la de mayor ganancia en enero (23\%).

El $50 \%$ de las pérdidas en julio y el $65 \%$ de las ganancias en enero se dan a través de las aberturas. Si discriminadas según modo de transferencia térmica, las ocurridas por infiltración superan en $3 / 4$ a la transferencia por conducción (Figura 5).

\section{HIPÓTESIS DE CÁLCULO}

Mejorar los niveles de retardo, o sea, bajar en horas el nivel de pérdidas en invierno y dispersión en verano, replanteando el nivel de transmitancia térmica de la envolvente en muros y cubierta, y de aislación e infiltración de las aberturas, permite la corrección de los ítems de mayor impacto "calefacción y refrigeración" y conservar las condiciones térmicas beneficiosas para el confort humano al mínimo requerimiento energético.

Contrastar esta hipótesis, lleva a analizar en qué grado el proyecto original existente se pensó en términos de sustentabilidad. Luego de calcular y verificar condiciones de aislación y resistencia térmica, según norma IRAM 11601 (2002) e IRAM 11549 (2002) -exigencias consideradas en Ley Provincial 13059 (2003)-, se simulan 3 (tres) variantes adicionales. Una del tipo de construcción masiva llamada "convencional"; otra en que la vivienda existente es mejorada en etapa de post ocupación retrofit; y una última en la que, en etapa de proyecto arquitectónico, se toman decisiones a fin de alcanzar la mejor EE y el menor impacto ambiental ex ante.

\section{CONVENCIONAL}

- Muros generales: Revoque interior $2 \mathrm{~cm}$, ladrillo hueco cerámico $18 \mathrm{~cm}$, revoque exterior $3 \mathrm{~cm}$ sin aislación. Valor $K$ (conductividad térmica) $=1,54 \mathrm{~W} / \mathrm{m}^{2} \mathrm{~K}$.

- Cubierta: Terminación con yeso $2 \mathrm{~cm}$, losa de hormigón armado de $12 \mathrm{~cm}$ sin aislación, membrana asfáltica $0,03 \mathrm{~cm} . \mathrm{K}=4,53 \mathrm{~W} / \mathrm{m}^{2} \mathrm{~K}$.

- Aberturas: Vano de abertura un 50\% mayor al existente. Ventana marco aluminio, vidrio simple, hoja de abrir, sin protección solar. $K=5,8 \mathrm{~W} / \mathrm{m}^{2} \mathrm{~K}$.

- Equipamiento: Igual a "existente".

\section{RETROFIT}

A la existente se adiciona o recambia:

- Muro al exterior: Adic. Placa termoaislante revestida $3 \mathrm{~cm} . K=0,55 \mathrm{~W} / \mathrm{m}^{2} \mathrm{~K}$.

- Cubierta: Adic. Lana de vidrio $8 \mathrm{~cm} . K=0,34 \mathrm{~W} / \mathrm{m}^{2} \mathrm{~K}$.

- Aberturas: Recambio ventana marco PVC y DVH $4+16+4$, de abrir, cortina de enrollar PVC. $K=1,91$ $W / m^{2} K$.

- Equipamiento: Igual a "existente"

\section{EX ANTE}

Nueva envolvente:

- Muro al exterior: Placa de yeso 1,5cm, cámara de aire $7 \mathrm{~cm}$, panel termoaislante PUR $10 \mathrm{~cm}$, terminación de chapa al exterior. $K=0,26 \mathrm{~W} / \mathrm{m}^{2} \mathrm{~K}$.

- Cubierta: Cielorraso suspendido de placa de yeso $1,5 \mathrm{~cm}$, capa de aire no ventilada $10 \mathrm{~cm}$, panel termoaislante PUR para techos $10 \mathrm{~cm} . K=0,25 \mathrm{~W} / \mathrm{m}^{2} \mathrm{~K}$.

- Aberturas: Ventana marco PVC y DVH 4+16+4, hoja de abrir, cortina de enrollar PVC. $K=1,91 \mathrm{~W} / \mathrm{m}^{2} \mathrm{~K}$.

- Equipamiento: Reducido (4 calefactores $1500 \mathrm{kcal} / \mathrm{h}$.) 
Transición energética argentina. El nuevo estándar de eficiencia energética en la evaluación de la vivienda social. Caso de estudio: Vivienda de Barrio Papa Francisco Ernesto Kuchen, Daniel Kozak

HS Revista Hábitat Sustentable Vol. 10, №. 1. ISSN 0719-0700 / Págs. $44-55$

\section{A $0-40$}

\section{B $40-80$}

Ex ante $-49 \mathrm{kWh} / \mathrm{m}^{2} . \mathrm{a}$

Retrofit - $68 \mathrm{kWh} / \mathrm{m}^{2} . \mathrm{a}$

\section{C $80-120$}

D) $120-180$

Existente $-132 \mathrm{kWh} / \mathrm{m}^{2} . \mathrm{a}$

\section{E $180-240$}

F $240-320$

Convencional $-281 \mathrm{kWh} / \mathrm{m}^{2} . \mathrm{a}$

\section{$\mathbf{G}>320 \mathrm{kWh} / \mathrm{m}^{2} \cdot \mathrm{a}$}

Figura 6. Etiqueta de Eficiencia Energética según IRAM 11900:v.2017. Valores IPE. Fuente: Elaboración de los autores.

Índice de Prestaciones Energéticas IPE [kWh Prim. $/ \mathrm{m}^{2}$.año]

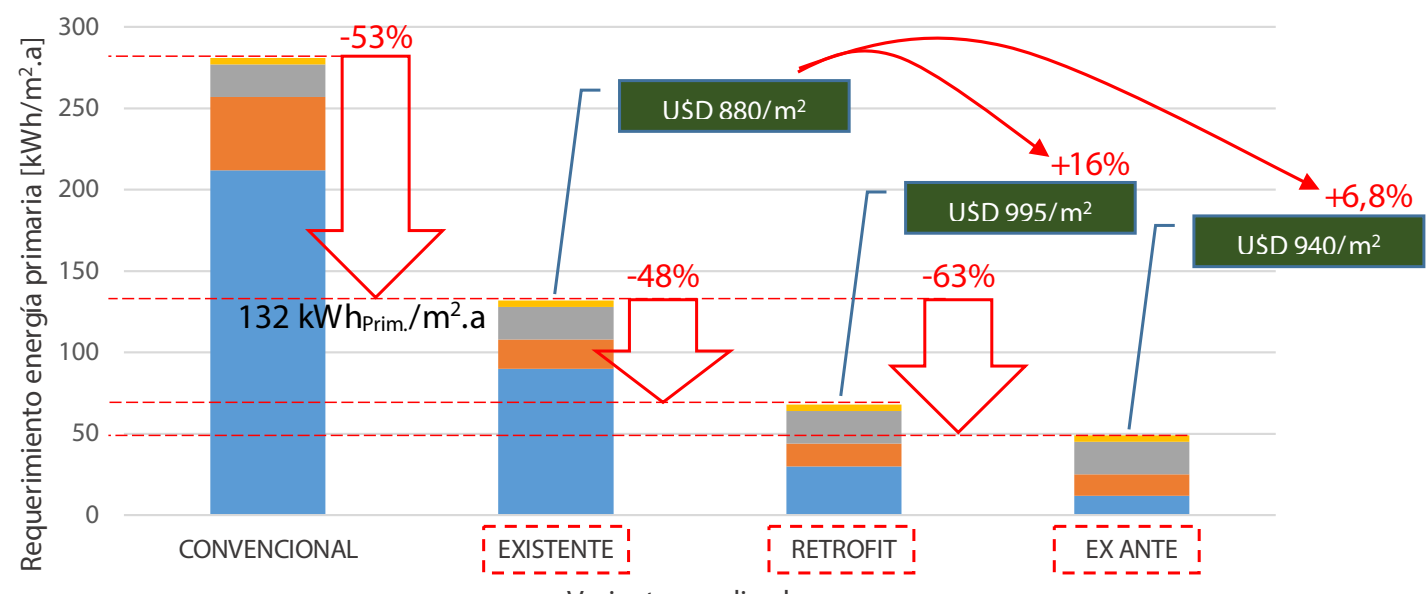

Variantes analizadas

$$
\text { - Calefacción } \quad \text { Refrigeración Producción acs } \quad \text { Iluminación }
$$

Figura 7. Requerimientos energéticos primarios por variante y año. Fuente: Elaboración de los autores. 


\begin{tabular}{|c|c|c|c|c|c|c|c|c|c|}
\hline Variante & $\begin{array}{l}\text { IPE [kWh } / \\
\text { m².a] }^{2} \text { ' }\end{array}$ & Sup. $\left[\mathrm{m}^{2}\right]$ & $\begin{array}{l}\text { Energía } \\
\text { [kWh/a] }\end{array}$ & $\begin{array}{l}\text { Costo año } \\
\text { [\$] }\end{array}$ & $\begin{array}{l}\text { Constr. } \\
\text { [U\$D/m²] }\end{array}$ & $\begin{array}{l}\text { Inversión } \\
\text { [U\$D/Un.] }\end{array}$ & $\begin{array}{l}\text { Inversión } \\
\text { [\$/Un.] }\end{array}$ & $\begin{array}{l}\text { Inv. Tec. [\$/ } \\
\text { Un.] }\end{array}$ & $\begin{array}{l}\text { Amortización } \\
\text { [años] }\end{array}$ \\
\hline Existente & 132 & 78,6 & 10375,2 & 30243,708 & 880,0 & $69.168,0$ & $5.083 .848,0$ & 0,0 & \\
\hline Retrofit & 68 & 78,6 & 5344,8 & 15580,092 & 995,0 & $78.207,0$ & $5.748 .214,5$ & $664.366,5$ & 45,3 \\
\hline Ex ante & 49 & 78,6 & 3851,4 & 11226,831 & 940,0 & $73.884,0$ & $5.430 .474,0$ & $346.626,0$ & 18,2 \\
\hline
\end{tabular}

Tabla 2. Comparación de costos energéticos versus costos económicos de las variantes retrofit y ex ante, respecto de la variante "existente". Fuente: Elaboración de los autores. Referencias divisa USD1,00/\$73,5 al 01/07/2020, Banco de la Nación Argentina.

De la simulación de las variantes propuestas, se obtienen los IPE, por variante y los valores se plasman en la etiqueta según IRAM 11900:v2017, en categorías (Figura 6). La variante "existente", con $132 \mathrm{kWh}$ Prim. $/ \mathrm{m}^{2} . \mathrm{a}$, se ubica en término medio, corresponde a categoría D, y llega a ser un $53 \%$ más eficiente que la variante "convencional", con 281 kWh/m².año (Categoría G). Las mejoras incorporadas a variante "existente", conducen un incremento en EE, logrando un IPE en variante retrofit de $68 \mathrm{kWh}$ Prim. $/ \mathrm{m}^{2}$. año y $49 \mathrm{kWh}$ Prim. $/ \mathrm{m}^{2}$. año en variante ex ante, ambas categorizadas " $\boldsymbol{B}^{\prime \prime}$ en la escala de EE.

\section{IMPACTO ECONÓMICO}

La Figura 7 muestra una evaluación general del costo de construcción [U\$D/m²] en relación al beneficio energético según variantes analizadas, considerando los ítems calefacción, refrigeración, ACS e iluminación en $\left[\mathrm{kWh} \mathrm{h}_{\text {prim }} . / \mathrm{m}^{2}\right.$.a.]. De aplicar mejoras, las variantes retrofit y ex ante llegan a ser, respectivamente, un $48 \%$ y un $63 \%$ más eficientes que la variante "existente". Las estrategias de aislación de la envolvente y recambio de aberturas logran ser efectivas. Para bajar el IPE en estas últimas deberá implementarse tecnología en renovables.

El análisis del costo $\left[U \$ \mathrm{D} / \mathrm{m}^{2}\right]$, en cuanto al beneficio energético $\left[\mathrm{kWh} \mathrm{h}_{\text {Prim. }} . / \mathrm{m}^{2}\right.$.año], indica que la variante retrofit representa incrementos del $16 \%$ por encima de la variante "existente". La variante ex ante llega a ser más conveniente económica y energéticamente por significar incrementos del 6,8\%, por encima de la "existente", y requerir solo $49 \mathrm{kWh}$ Prim. $/ \mathrm{m}^{2}$. año (Figura 7 ).

A modo de síntesis, la Tabla 2 presenta valores de prestaciones (IPE) y costos energéticos anuales según variante (Ref. 2,915 \$/kWh, T1-R3, residencial hasta 400 kWh, EDENOR) y su relación con los costos de construcción [U\$D/Unidad] que se muestran en la Figura 7.

A fin de estimar un período de amortización de la inversión, se computan a valores locales los costos de inversión en tecnología [\$/Un.], respecto a los ahorros anuales potenciales por variante [kWh/año]. Ello hace posible determinar que de perseguir la variante retrofit, el horizonte de amortización será de 45,3 años y de optarse por la variante ex ante, será de 18,2 años.

Debe aclararse que este análisis no contiene la afectación que sufriría el precio de la energía en el tiempo, calculado por Marinozzi (2020) en base a un factor de crecimiento de precio monómico anual de 0,672. De esta forma, es difícil afirmar una hipótesis de la evolución de precio de la energía a futuro y, por tanto, las estimaciones de la amortización son lábiles.

\section{IMPACTO AMBIENTAL ASOCIADO}

El impacto ambiental asociado es directamente proporcional al consumo energético y dependerá del vector energético involucrado (ver punto 3.2). Según el inventario nacional de gases de efecto invernadero (Medio Ambiente y Desarrollo Susutentable-MAyDS, 2017), el sector residencial impacta en $5^{\text {to }}$ lugar, después de ganadería, transporte, silvicultura y generación de electricidad, con 28,41 MtCO2eq/año, lo que representa el $7,7 \%$, sin considerar impacto de aguas residuales y residuos sólidos urbanos (3,8\%). En relación al consumo energético, el impacto ambiental del mix energético argentino, al año 2018, es de 412 gCO2eq/kWh. De las variantes, la "existente" emite 54 kgCO2eq/año.

De considerar que el impacto de 1700 viviendas en Papa Francisco en 80 años (vida útil), podría ser 0,007 MtCO2eq, reducir el impacto asociado al consumo de recursos constituye una tarea fundamental para el desarrollo sustentable e inclusivo en la integración de los barrios en un plan de transición energética.

\section{CONCLUSIONES}

La distribución de recursos energéticos planificada se convierte en leitmotiva la hora de garantizar accesibilidad y equidad en el uso del servicio energético, lo cual, sin lugar a dudas, es el motor de una transición energética obligada. 
La posibilidad de referenciar valores admisibles de demanda de energía por superficie y año en la comparación, así como el hacer visibles los beneficios energéticos, económicos y ambientales traducidos en una etiqueta de EE energética (IRAM 11900, 2017), contribuyen con el proceso de concientización de una población en formación. La norma representa un cambio de paradigma para la construcción sustentable.

La Ley 13.903 (2017) de Santa Fe promueve su impulso a que inmuebles clasificados con categoría " $A$ " reciban la bonificación del 30\% del impuesto inmobiliario urbano. Se espera que una vivienda que acceda a una etiqueta con Clase de Eficiencia Energética CEE, se registre en la escritura de dominio y se traslade al Registro General de la Propiedad a fin de otorgar valor a la unidad y motivación a propietarios e inquilinos.

Las resoluciones efectuadas en la concepción de los proyectos son las más determinantes y las que ofrecen un mejor balance en la relación costo-beneficio. A medida que avanza el proceso proyectual y la construcción se van cerrando posibilidades de modificar a conciencia. El Índice de Prestaciones Energética (IPE), como referencia para la comparación con valores de referencia, constituye una herramienta clave a la hora de proyectar inmuebles desde cero.

La unidad del Barrio Papa Francisco responde a mejores criterios de sustentabilidad respecto de una variante convencional de uso masivo, aunque de haberse iniciado la evaluación de los beneficios en su fase inicial, estaríamos en presencia de una solución constructiva un $63 \%$ más eficiente con un impacto asociado la mitad más bajo y apenas un 6,8\% más onerosa, y dejando una impronta clave en planificación del ambiente construido.

\section{AGRADECIMIENTOS}

Se agradece el financiamiento del Consejo Nacional de Investigaciones Científicas y Técnicas de Argentina, al Instituto de Doble Dependencia IRPHa-UNSJ-CONICET, al CEUR-CONICET y al CIHE-FADU-UBA. También a la Arq. Eliana Ghia y la Arq. Natalia Felder en el análisis de datos y evaluaciones energéticas, y al Dr. Martín Motta y al Instituto de la Vivienda de la Ciudad de Buenos Aires por el aporte de información.

\section{REFERENCIAS BIBLIOGRÁFICAS}

Alonso Frank, A. y Kuchen, E. (2017). Validación de la herramienta metodológica de Alonso Frank \& Kuchen para determinar el indicador de nivel de eficiencia energética del usuario de un edificio residencial en altura, en San Juan-Argentina. Revista Hábitat Sustentable, 7(1), 6-13.
Brent, A. C. y Petrick, W. (2007). Environmental impact assessment during project execution phases: towards a stage-gate project management model for the raw materials processing industry of the energy sector. Impact Assessment and Project Appraisal, 25(2), 111-122. https://doi.org/10.3152/146155107X205832

Chevez, P. (2017). Construcción de escenarios urbano-energéticos a partir de la implementación de estrategias de eficiencia energética y energías renovables en el sector residencial. Tesis doctoral. Universidad Nacional de Salta. Facultad de Ciencias Exactas Doctorado en Ciencias - Área Energías Renovables.

Etiquetado de Viviendas (2020). Aplicativo Informático Nacional 2020. Recuperado de http://etiquetadoviviendas.energia.gob.ar/

Evans, J. M., De Schiller, S. y Kozak, D. (2015) - Auditorías de comportamiento térmico y eficiencia energética en edificios con acondicionamiento natural. En: S. de Schiller y J.M. Evans (Eds.), Eficiencia Energética en el Hábitat Construido (pp. 191-202). Buenos Aires: CIHE-FADU-UBA.

Fernández, R. (coord.). (2019). Transición Energética 2050. Hacia una visión compartida de la transición energética argentina al 2050: propuesta de objetivos y metas. (Edición: J. Dumas y D. Ryan). Buenos Aires: Centro de Estudios de la Actividad Regulatoria Energética (UBA), Instituto Tecnológico de Buenos Aires (ITBA), Fundación AVINA, Programa de las Naciones Unidas para el Desarrollo, Secretaría de Gobierno de Energía.

Instituto de Vivienda de la Ciudad (IVC). (2016). Integración de los barrios. Recuperado de https://vivienda.buenosaires.gob.ar/\#top

IRAM 11549 (2002). Instituto Argentino de Normalización y Certificación. Aislamiento Térmico en Edificios- Vocabulario. Buenos Aires, Argentina.

IRAM 11601 (2002). Instituto Argentino de Normalización y Certificación. Aislamiento Térmico de Edificios: Métodos de Cálculo. Buenos Aires, Argentina.

IRAM 11603 (2012). Instituto Argentino de Normalización y Certificación. Acondicionamiento térmico en Edificios: Clasificación Bioambiental de la República. Buenos Aires, Argentina.

IRAM 11900 (2017). Instituto Argentino de Normalización y Certificación. Prestaciones energéticas en viviendas. Método de cálculo y etiquetado de eficiencia energética. Buenos Aires, Argentina.

Kozak, D., Evans, J., Adamo, G., Abálsamo, D. y Romanello, L. (2017). Sustentabilidad en viviendas de escala intermedia en Buenos Aires. Revista Summa, 155, 102-110.

Kuchen, E., Plesser, S. y Fisch, M.N. (2012). Eficiencia energética y confort en edificios de oficina. El caso alemán. Revista Internacional Hábitat Sustentable, 2(2), 34-44.

Ley No 13.059 (2003). Poder Legislativo de la Provincia de Buenos Aires. Acondicionamiento térmico de edificios de la Provincia de Buenos Aires. Cámara empresaria de desarrolladores urbanos de la República Argentina. Buenos Aires, 9 de abril de 2003.

Ley No 13.903 (2017). Poder Legislativo de la Provincia de Santa Fe. Etiquetado de eficiencia energética de inmuebles destinados a vivienda. Cámara de Diputados de la Provincia de Santa Fe, 1 de octubre de 2017 
Li, F., Trutnevyte, E. y Strachan, N. (2015). A review of sociotechnical energy transition (STET) models". Technological Forecasting and Social Change, 100, 290-305. https://doi. org/10.1016/j.techfore.2015.07.017

Marinozzi, E. (2020). Informe Mensual CAMMESA. Principales Variables del Mes. Gerencia Análisis y Control Global. Mayo 2020. Recuperado de https://portalweb.cammesa.com/default.aspx

Riavitz, L., Zambon, H. y Giuliani, A. (2015). La matriz energética argentina y la restricción externa. Cuadernos de Investigación. Serie Economía, (5), 110-141.

Risuelo, F. (2010). Certificados de eficiencia energética en edificios. Resumen ejecutivo. Buenos Aires: FODECO.

Secretaría de Energía de la Nación (SEN). (2020) Eficiencia Energética: Programas. SEN 2020. Recuperado de https://www. argentina.gob.ar/produccion/energia

Secretaría de Medio Ambiente y Desarrollo Sustentable (MAyDS) (2017). Inventario de Gases de Efecto Invernadero de Argentina. Recuperado de https://www.argentina.gob.ar/sites/default/files/ inventario-nacional-gei-argentina.pdf 